\title{
Basic Research Needs for
}

\section{Transformative Manufacturing}




\section{Transformative Manufacturing-Fundamental science for innovative manufacturing approaches and products}

Manufacturing is central to the nation's prosperity and security. Manufacturing currently represents about 12\% of the gross domestic product, provides nearly 13 million jobs, and accounts for about 25\% of energy use. The nation's economy relies heavily on wide-ranging manufacturing sectors — all of which share common challenges including data issues, lack of physics and chemistry-based models across scales, and resource constraints in a global environment. Furthermore, there are many hurdles that must be overcome to move basic science innovations to market. Addressing broad-ranging challenges demands a basic-science strategy that underpins applied research activities. This strategy would accelerate innovation and transform manufacturing.

A Basic Research Needs workshop for Transformative Manufacturing was held in March 2020. The focus of the workshop was to identify the basic science research priorities that could accelerate innovation to transform manufacturing in the future. This was the first workshop of its kind to examine how basic energy science can drive manufacturing forward and innovate new ways to manufacture goods. Five Priority Research Directions were identified that address these science challenges: (1) innovative synthetic approaches to enable scalable assembly of matter, (2) computational methods and theoretical models to transform how manufacturing processes are controlled, (3) new characterization tools that can handle the necessary complexity, scales, and processing speeds to meet manufacturing needs, (4) new science to address opportunities relevant to sustainable and energyefficient manufacturing, and (5) foundational approaches to co-design of materials, process, and products. The full workshop report will be posted at http://science.osti.gov/bes/Community-Resources/Reports.

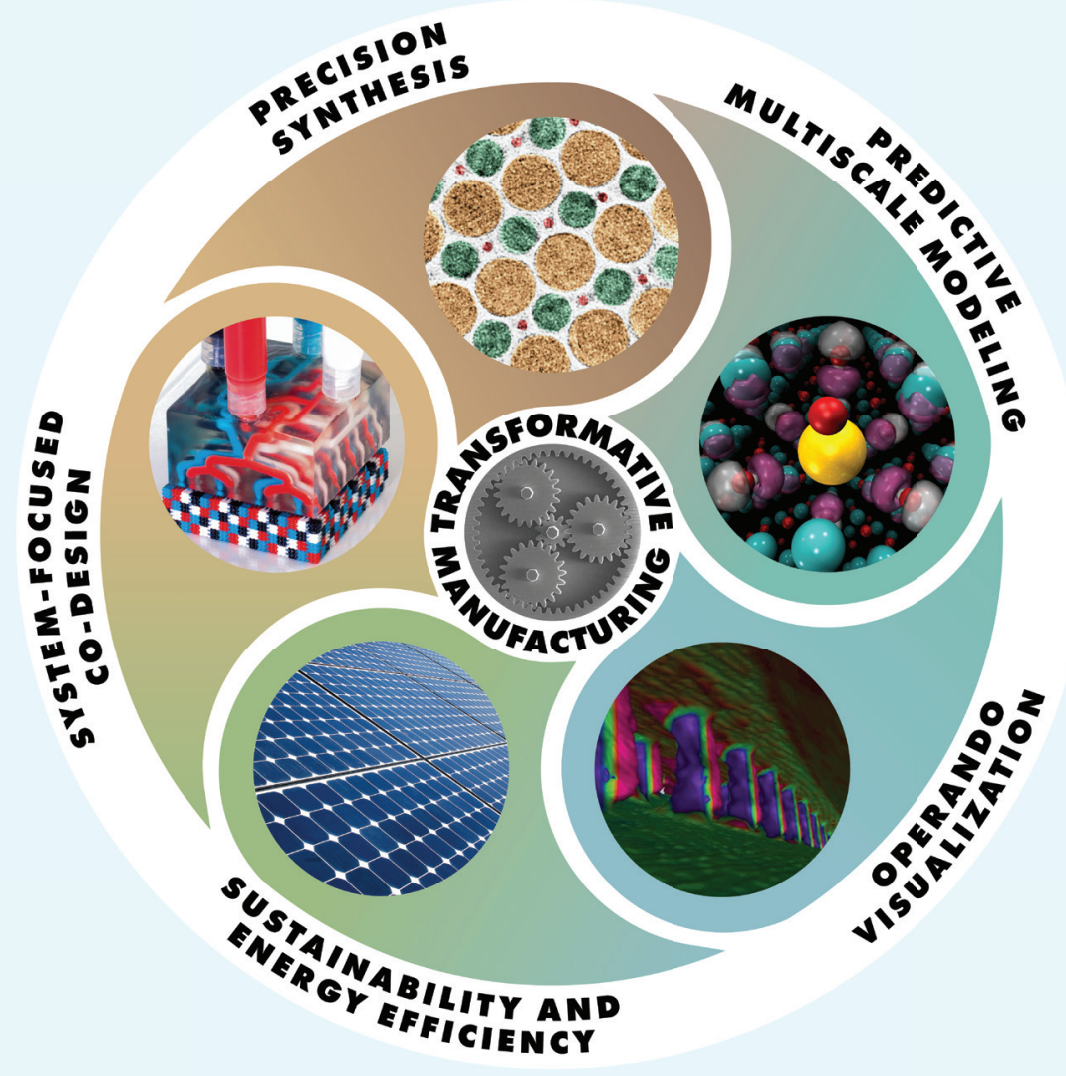

Transformations in manufacturing require a multidisciplinary understanding to discover new approaches that fully integrate modeling, data analytics, and characterization and reduce lifecycle energy and resource impacts. These five priority research directions comprise fundamental science strategies to accelerate innovation and solve industry's toughest challenges in manufacturing. 


\section{Priority Research Directions}

- Achieve precise, scalable synthesis and processing of atomic-scale building blocks for components and systems

Key Questions: What are the mechanisms needed for manufacturing multiscale, atomically and molecularly precise materials? How can basic research uncover structure-function relationships across multiple scales in components and systems? How can chemical processes readily be scaled from laboratory results?

Innovations that enable precise and scalable synthesis and processing will accelerate the transition from current manufacturing methods to new paradigms for creating unparalleled structures and functions. Application-specific materials with unprecedented performance at manufacturing scale will emerge from targeted synthesis and processing of building blocks, components, and systems that are precisely controlled at the atomic scale.

- Integrate multiscale models and tools to enable adaptive control of manufacturing processes

Key Questions: What are the frameworks required to model, monitor, and ultimately control manufacturing processes that tightly couple physics and chemistry across scales? How can complex multiscale models be translated to fast surrogate models for process control?

Linkages between small-scale physics and chemistry to macro-scale nonequilibrium processes and component performance are not fully understood, limiting the achievable precision and functionality of products. A combination of multiscale modeling, in situ diagnostics, and an online decision-making framework is needed to realize adaptive manufacturing processes and guarantee component qualification.

- Unravel the fundamentals of manufacturing processes through innovations in operando characterization

Key Questions: How can manufacturing processes and products be "visualized" at the atomic level, in real time, and under operating conditions to reveal the intricate details of underlying physical or chemical events? How can these insights be used in control schemes that inform decision making?

In 21st century manufacturing, many processes are still practiced as "art" instead of science. Frequently, there is insufficient fundamental understanding to tailor and control materials and processes so that they perform exactly as desired, with minimum energy consumption and maximum efficiency. Operando characterization — direct visualization and characterization under actual manufacturing conditions - will provide the knowledge needed to transform the science of manufacturing.

\section{- Direct atom and energy flow to realize sustainable manufacturing}

Key Questions: What are the methodologies to achieve atom and energy efficiency for sustainable manufacturing? How can science enable adaptive and resilient manufacturing across scales to exploit renewable or recycled feedstocks?

Sustainable manufacturing requires localizing energy delivery and directing atom- and energy-efficient chemical and materials processes. Synergistically using diverse forms of energy (e.g., electrical, thermal, radiative, and mechanical) coupled with understanding phenomena across length scales would enable sustainable, high-efficiency processes. Scientific advances could support the design of circular feedstocks that minimize waste and reduce the use of critical materials in existing and future chemicals and materials, moving toward resiliency.

\section{- Co-design materials, processes, and products to revolutionize manufacturing}

Key Questions: How can bottom-up scientific discovery be combined with top-down system-focused design to identify new and efficient manufacturing modalities? What new approaches will allow the control of matter in the presence of impurities and/ or nonequilibrium states? How can science enable multiple performance objectives to be achieved simultaneously for complex, multicomponent processes?

Co-design is a paradigm that provides scientific foundations for the creation of new materials, chemical processes, or systems by addressing the ubiquitous manufacturing challenge of simultaneously satisfying multiple performance objectives. Exciting opportunities exist to meet this challenge with new approaches integrating predictive modeling and experimental data with system resiliency, circularity, and operability. Doing so will enable the navigation of the nearinfinite range of possible designs to identify inherently resilient systems. 


\section{Summary}

Fundamental science plays a pivotal role in how products are made and enables realization of products not yet imagined. The Priority Research Directions outlined here constitute a basic science strategy that underpins applied technology research. To transform manufacturing, fundamental advances in synthesis, processing, modeling, operando characterization, and validation are needed. As manufacturing processes become increasingly data driven and fully networked, integration of autonomous sensing and control will enable greater productivity and competitiveness. Finally, co-design will tackle data, control, and design across components, delivering multiple system-level performance criteria simultaneously. Research based on these priorities will lay the scientific foundation to go beyond incremental improvements to create new, transformative technologies for manufacturing that are energy efficient and sustainable.

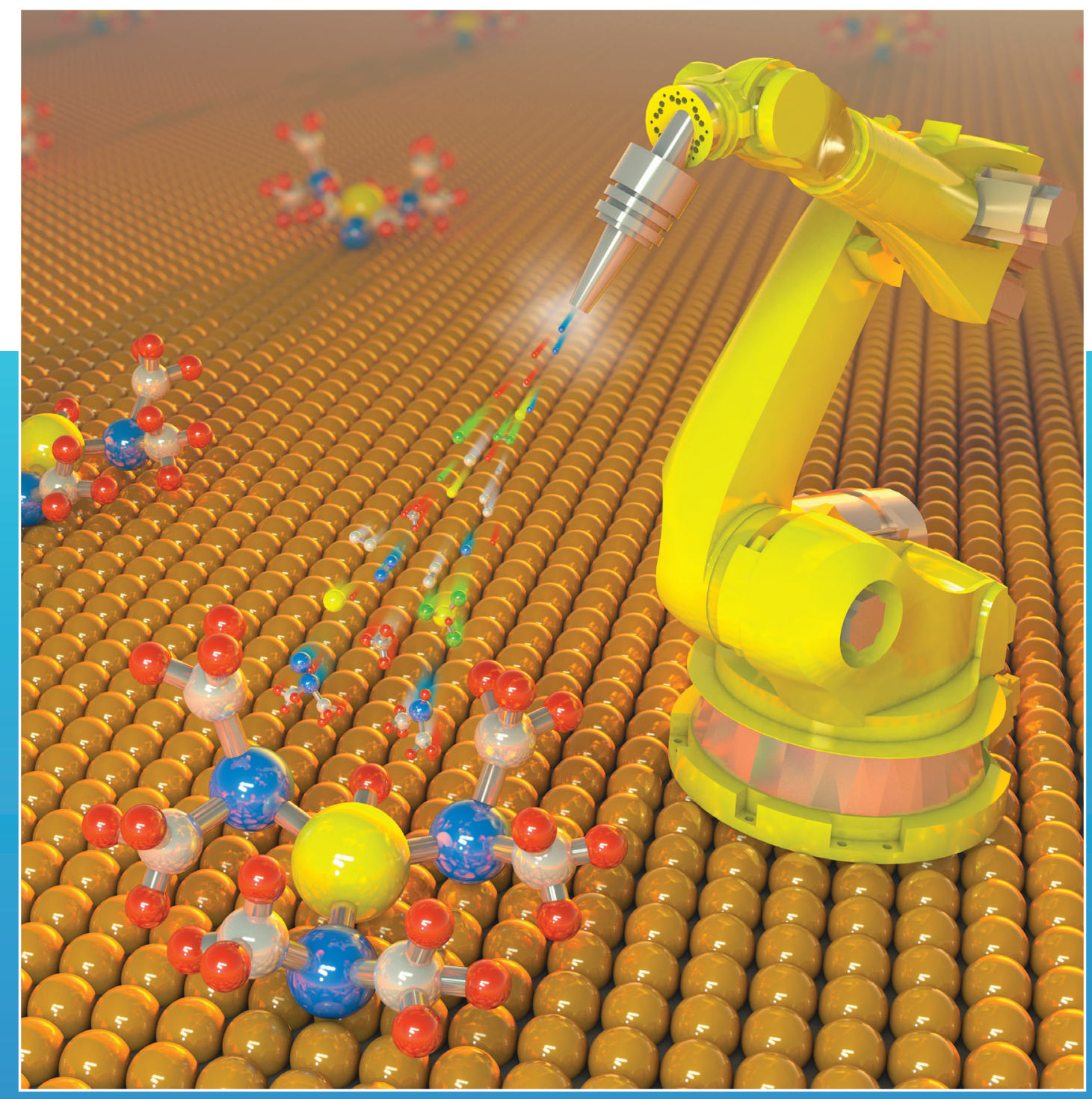

Cover and back images courtesy of Oak Ridge National Laboratory.

Sidebar images courtesy of Oak Ridge National Laboratory, Argonne National Laboratory, and reprinted with permission from the American Chemical Society and Springer/Nature.

DISCLAIMER: This report was prepared as an account of work sponsored by an agency of the United States government. Neither the United States government nor any agency thereof, nor any of their employees, makes any warranty, express or implied, or assumes any legal liability or responsibility for the accuracy, completeness, or usefulness of any information, apparatus, product, or process disclosed, or represents that its use would not infringe privately owned rights. Reference herein to any specific commercial product, process, or service by trade name, trademark, manufacturer, or otherwise does not necessarily constitute or imply its endorsement, recommendation, or favoring by the United States government. 